\title{
Monte Carlo simulation of joint density of states in one-dimensional Lebwohl-Lasher model using Wang-Landau algorithm
}

\author{
Kisor Mukhopadhyay ${ }^{a}$, Nababrata Ghoshal ${ }^{b}$ and Soumen Kumar Roy ${ }^{c, *}$ \\ ${ }^{a}$ Department of Physics, Sundarban Mahavidyalaya, \\ Kakdwip, South 24 Parganas, West Bengal, INDIA \\ ${ }^{b}$ Department of Physics, Mahishadal Raj College, \\ Mahishadal, Midnapore (East), West Bengal, INDIA \\ ${ }^{c}$ Department of Physics, Jadavpur University, \\ Kolkata - 700 032, INDIA
}

\begin{abstract}
Monte Carlo simulation using the Wang-Landau algorithm has been performed in an one-dimensional Lebwohl-Lasher model. Both one-dimensional and two-dimensional random walks have been carried out. The results are compared with the exact results which are available for this model.
\end{abstract}

PACS: 61.30.-v, 64.70.Md

Keywords: Monte Carlo, joint density of states

${ }^{*}$ E-mail addresses: ${ }^{a}$ kisor_m@yahoo.com, ${ }^{b}$ ghoshaln@yahoo.co.in, ${ }^{c}$ skroy@phys.jdvu.ac.in, 


\section{Introduction}

The Wang-Landau (WL) approach [1] in Monte Carlo (MC) simulation, introduced in 2001, has since been applied to different areas of statistical physics. While proposing the algorithm the authors have demonstrated the application of the algorithm to systems with discrete energy levels (like the Ising system, Potts model, spin-glass etc.). Over the past few years several authors [2, 3, 4] have reported the application of the WL method to systems with continuous energy spectrum. The common feature of these simulations is the expected discretization of the energy range which has been investigated i.e. the division of the energy range into a number of bins and the subsequent application of the ideas of Wang and Landau. It may however be noted that in majority of these simulations a random walk in energy space alone was conducted. Relatively few papers [5, 6] have so far appeared where a two dimensional random walk has been performed in a continuous system.

It has generally been noted that even for a modest system size, the simulation of joint density of states, where the variables for instance are energy and the order parameter, (both quantities being continuously variable), the computational time necessary for the implementation of the WL algorithm is very large. It may further be noted that till now no work has been published regarding the errors involved in the WL-simulation in such systems. Also, no simulation has so far been reported where comparison has been made with the exact results available (as is usually done for discrete systems with the two dimensional Ising model). The reason for this may of course be attributed to the non-availability of results of exact calculation for any non-trivial system having a continuous energy spectrum.

The present work has been intended to fill the gap in the availability of adequate information in this direction to some extent. The system we have chosen for this purpose is an one-dimensional array of three-dimensional spins $(d=1, n=3$, where $d$ is the system dimensionality and $n$ is the spin dimensionality) interacting with nearest neighbours via a potential $-P_{2}\left(\cos \theta_{i j}\right)$, where $P_{2}$ is the second Legendre polynomial and $\theta_{i j}$ is the angle between the nearest neighbour spins $i$ and $j$, (the coupling constant in the interaction has been set to unity). This model, known as the Lebwohl-Lasher (LL) model [7], is the lattice version of the Maier-Saupe (MS) model [8, 9, 10] which describes a nematic liquid crystal in the mean field approximation.

The one-dimensional LL model $(d=1, n=3)$, which of course does not have any ordered 
state and consequently can not exhibit any finite temperature order-disorder transition, has been solved exactly by Vuillermot and Romerio [11, 12] in 1973, using a group theoretical method. We decided to choose this simple model to apply and test the performance of the WL algorithm for simulation of joint density of states so that a comparison can be made with the exact results available.

We have performed WL simulation using one-dimensional random walk, where the visits are confined to the energy space alone, and also a two-dimensional random walk where the two dimensional space spanned is the energy-order parameter space as well as energycorrelation function space. The partition function $Z$ can directly be computed as a function of temperature from a knowledge of the density of states, $g(E)=\ln \Omega(E)$, using the relation

$$
Z(T)=\sum_{E} \Omega(E) e^{-\beta E}
$$

where $\beta=1 / T$ (the Boltzmann constant has been set to unity). In the two dimensional walk one computes $\Omega(E, \phi)$ where $\phi$ is the order parameter of the system or a correlation function which are defined in a following section. The partition function can be computed from a knowledge of $\Omega(E, \phi)$ :

$$
Z(T)=\sum_{E} \sum_{\phi} \Omega(E, \phi) e^{-\beta E}
$$

The ensemble average of any function of $\phi$ at a temperature $T$ is given by,

$$
\langle f(\phi, T)\rangle=\frac{\sum_{E} \sum_{\phi} f(\phi) \Omega(E, \phi) e^{-\beta E}}{\sum_{E} \sum_{\phi} \Omega(E, \phi) e^{-\beta E}}
$$

We have computed $\ln Z$ from both 1 -d and 2 -d random walks and have compared the results obtained with those available from the exact results of Ref. [11, 12].

\section{The one dimensional Lebwohl-Lasher model and the exact results}

The Hamiltonian of the Lebwohl-Lasher model is given by

$$
H=-\sum_{\langle i, j\rangle} P_{2}\left(\cos \theta_{i j}\right)
$$


where $P_{2}$ is the second order Legendre polynomial and $\theta_{i j}$ is the angle between the nearest neighbour spins $i$ and $j$. The spins are three dimensional and headless, i.e. the system has the $\mathrm{O}(3)$ as well as the local $Z_{2}$ symmetry characteristic of a nematic liquid crystal. A vector order parameter is inadequate for the system and a traceless second rank tensor $\underline{\mathrm{Q}}$, as defined below, is used to describe the orientational order of the system [13]. One uses,

$$
Q_{i j}=\frac{1}{N} \sum_{t=1}^{N}\left(n_{i}^{t} n_{j}^{t}-\frac{1}{3} \delta_{i j}\right)
$$

where $n_{i}^{t}$ is the $i$-th component of the unit vector $\hat{n}$, which points along the spin at the site $t . N$ is the number of particles in the system. In the ordered state $\langle\underline{Q}\rangle$ is non-zero. In a coordinate system with the Z-axis points along the direction of molecular alignment (director) the matrix $\langle\underline{Q}\rangle$ is diagonal and for a uniaxial system,

$$
\langle\underline{\mathbf{Q}}\rangle=S\left(\begin{array}{ccc}
-1 / 3 & 0 & 0 \\
0 & -1 / 3 & 0 \\
0 & 0 & 2 / 3
\end{array}\right)
$$

where,

$$
S=\frac{1}{2}\left\langle\left(3 \cos ^{2} \theta^{t}-1\right)\right\rangle=\left\langle P_{2}\left(\cos \theta^{t}\right)\right\rangle
$$

where $\theta^{t}$ is the angle between a spin and the director.

MC simulations demonstrate that a three dimensional Lebwohl-Lasher model $(d=3, n=3)$ exhibits a weakly first order transition, characteristic of a nematic-isotropic transition which is available from the Maier-Saupe model of a nematic in the mean field approximation. On the other hand, for lattice dimensionality $d=2$ and 1 , no true long range order is expected since Mermin-Wagner theorem [14] predicts a fluctuation destruction of long range order. The $d=2$ LL model has been investigated by a number of authors [15, 16] and the system shows a behaviour qualitatively similar to the two dimensional XY model. A quasi-long range order has been observed in this system and this is believed to be related to the existence of topological defects in the system [17].

The one-dimensional Lebwohl-Lasher model has been simulated by [18] and has also been solved exactly [11, 12]. The system is known to be disordered at all finite temperatures and critical behaviour is expected only at $T=0$, which resembles an one-dimensional Ising model 
or the one dimensional Heisenberg model. The second rank spin-spin correlation function $\rho(r)$ is defined as

$$
\rho(r)=\left\langle P_{2}(\cos \theta(r))\right\rangle
$$

where $\theta(r)$ is the angle between two spins, $r$ lattice spacings apart. In the thermodynamic limit one would expect both $S$ and $\operatorname{Lim} r \rightarrow \infty \rho(r)$ to vanish whereas in finite systems because of finite size effects both quantities may appear to have small but finite values.

Vuillermot and Romerio [11, 12] presented an exact solution of the planar $(n=2)$ and spatial $(n=3)$ versions of the Lebwohl-Lasher model in one dimension $(d=1)$ for a nematic liquid crystal, without periodic boundary conditions. They also calculated the two-molecule correlation functions and have shown that these models do not exhibit any finite temperature order-disorder phase transition.

The partition function $Z_{N}(\tilde{K})$ for the $N$-particle system is given by

$$
Z_{N}(\tilde{K})=\tilde{K}^{-N / 2} \exp \left[\frac{2}{3} N \tilde{K}\right] D^{N}\left(\tilde{K}^{1 / 2}\right)
$$

where $\tilde{K}=3 / 2$ T. $D$ is Dawson function $[19$.

$$
D(x)=\exp \left[-x^{2}\right] \int_{0}^{x} d u \exp \left[u^{2}\right]
$$

The dimensionless internal energy $u_{N}(\tilde{K})$, the entropy $S_{N}(\tilde{K})$ and the specific heat $C_{N}(\tilde{K})$ are given by

$$
\begin{gathered}
\frac{2 U_{N}(\tilde{K})}{N}=1+\frac{3 \tilde{K}^{-1}}{2}-\frac{3}{2} \tilde{K}^{-1 / 2} D^{-1}\left(\tilde{K}^{1 / 2}\right) \\
\frac{S_{N}(\tilde{K})}{N}=\frac{1}{2}+\tilde{K}-\frac{1}{2} \tilde{K}^{1 / 2} D^{-1}\left(\tilde{K}^{1 / 2}\right)+\ln \left[\tilde{K}^{-1 / 2} D\left(\tilde{K}^{1 / 2}\right)\right]
\end{gathered}
$$

and

$$
\frac{2 C_{N}(\tilde{K})}{N}=1-\tilde{K}^{3 / 2}\left[\frac{\tilde{K}^{-1}}{2}-1\right] D^{-1}\left(\tilde{K}^{1 / 2}\right)-\frac{1}{2} \tilde{K} D^{-2}\left(\tilde{K}^{1 / 2}\right) .
$$

The correlation function is given by

$$
\rho_{N}(r)=\left[\frac{3}{4} \tilde{K}^{-1 / 2} D^{-1}\left(\tilde{K}^{1 / 2}\right)-\frac{3}{4} \tilde{K}^{-1}-\frac{1}{2}\right]^{r}
$$




\section{Computational details}

In the model we have investigated, spins can take up any orientation in the three dimensional space and the orientation of each spin is stored in terms of the direction cosines $\left(l_{1}, l_{2}, l_{3}\right)$. The starting configuration has always been chosen as a random one and to generate a new microstate, a randomly selected spin is chosen and each direction cosine of it is updated as $l_{i} \rightarrow l_{i}+p * r_{i}$ (for $\left.\mathrm{i}=1,2,3\right)$ where $p$ is a parameter to be chosen according to some criterion and $r_{i}$ is a random number between -1 to +1 . To preserve the unit magnitude of the spins, $\left(l_{1}, l_{2}, l_{3}\right)$ is always normalized.

The energy of the system in the LL model is a continuous variable and in one dimension $(d=1)$ it can have any value between $-L$ to $L / 2$. To have a discretization scheme for the implementation of the WL algorithm and for an one dimensional random walk in the energy space, we have chosen an energy range from $(-L$ to 0$)$ and divided this energy range into $M$ bins each having a width $d_{e}$.

We use $g\left(E_{i}\right)=\ln \Omega\left(E_{i}\right)$ where, $\Omega\left(E_{i}\right)$ is the number of micro-states corresponding to the $i$-th bin for which the mid-point has the value $E_{i}$. Initially we set all $g\left(E_{i}\right)(\mathrm{i}=1, \mathrm{M})$ to zero and the logarithm of the modification factor $\ln f$ is taken as 1 . Whenever a new microstate is generated by rotating a spin, the new system-energy and hence, the macrostate $\mathrm{j}$ is determined. Whether the move is accepted or not is decided according to the WL prescription [1] for the probability

$$
p_{i \rightarrow j}=\min \left(\frac{\Omega\left(E_{i}\right)}{\Omega\left(E_{j}\right)}, 1\right) .
$$

If the state $\mathrm{j}$ is accepted, we make $g\left(E_{j}\right)=g\left(E_{j}\right)+\ln f$ and $h\left(E_{j}\right)=h\left(E_{j}\right)+1$, where $h\left(E_{j}\right)$ is the histogram count. Otherwise we make $g\left(E_{i}\right)=g\left(E_{i}\right)+\ln f$ and $h\left(E_{i}\right)=h\left(E_{i}\right)$

+1 . This procedure is repeated for $10^{4} \mathrm{MC}$ sweeps (where one MC sweeps consists of $L$ attempted moves) and the flatness of the histogram is checked and the cycle is repeated till $90 \%$ flatness in the histogram is reached. This completes one iteration, following which we reduce the logarithm of the modification factor $\ln f \rightarrow \ln f / 2$, reset the histogram, and the whole procedure is repeated. For each lattice size we have continued with the iterations till $\ln f$ gets reduced to $10^{-9}$.

We have also calculated the quantity $S$ (Eq. (7)) which gives us the magnitude of the 
order parameter obtained from the largest eigenvalue of the ordering matrix defined in Eq. (5) and a two-dimensional random walk was performed in the $(E-S)$ space for this purpose. This is necessary if one intends to determine quantities other than those like free energy, entropy, specific heat etc. which are directly related to energy, and is particularly useful, if one needs to compute, for instance, the variation of the order parameter in presence of an external field. It is also possible to calculate the order parameter from an one-dimensional walk in energy space alone, as has been demonstrated in ref. [4] by Jayasri et. al. for a liquid crystalline system (Here one uses the procedure of the so called histogram 'unweighting' and 'reweighting'). But for a more accurate calculation of the order parameter (or the correlation function), it is perhaps a good idea, to generate a two dimensional walk in the $(E-S)$ space (or in the $(E-\rho(r))$ space) and check its flatness. For a given value of the energy of the system, the order parameter, $S$ has a distribution over a certain range of values. The whole range of $S$ is 0 to 1 and in order to perform the two dimensional random walk in the energyorder parameter $(E-S)$ space we divide the two dimensional space into $M \times N$ bins. We represent by $d_{\phi}$ the bin-width of the bins involving the parameter other than energy in the two-dimensional walk. Each microstate will now correspond to a macrostate labelled by the indices $i$ and $j$ and the acceptance probability given by Eq. (14) is now modified to

$$
p_{i j \rightarrow k l}=\min \left(\Omega\left(E_{i}, S_{j}\right) / \Omega\left(E_{k}, S_{l}\right), 1\right)
$$

along with an appropriate modification of the procedure described after Eq. (14) for the two-dimensional random walk. Here, for instance, $\Omega\left(E_{i}, S_{j}\right)$ is the density of states for the $i$-th energy and $j$-th order parameter bin.

A two-dimensional random walk in the $(E-S)$ space is a lot more expensive in terms of the CPU time than an one-dimensional walk in the energy space alone. The problem is particularly severe in a system with continuous energy and becomes worse as the lattice size increases. However, this ensures a much more uniform sampling of the order parameter bins that correspond to a particular energy bin and this improves the overall statistics of the work. It may be pointed out that it is impossible to arrive at a flat histogram in the $(E-S)$ space if one attempts to visit the entire energy and order parameter ranges accessible to the system. For an one-dimensional walk one normally faces a problem in that, it takes a relatively long time to visit the lowest energy levels and this increases with the increase in system size. For a two-dimensional walk the possibility of uniformly visiting the entire 
rectangular $(E-S)$ space is unphysical and one must have a prior knowledge of the range of $S$-bins which are likely to be visited while the system energy has a given value $E_{i}$. Our method of simulating the two dimensional random walk has resemblance to the work of Troster and Dellago [20], who have applied the WL algorithm to evaluate multidimensional integrals of sharply peaked functions. Our modified approach is elaborated in the following paragraph.

We have first mapped the $(E-S)$ space which costed us $35 \times 10^{6}$ sweeps (to be called the pre-production run). The idea is to determine the minimum $\left(S_{\min }^{i}\right)$ and maximum $\left(S_{\max }^{i}\right)$ values of the $S$-bins which are visited while the system energy is $E_{i}$ for $\mathrm{i}=1, \mathrm{M}$. We observe that there are always some $S$-bins within the range $\left(S_{\min }^{i}, S_{\max }^{i}\right)$ for each $E_{i}$, where either no sampling or very low sampling takes place during the pre-production run. We therefore checked the histograms of the $\left(E_{i}, S_{j}\right)$ bins in the mapped region of the two-dimensional space and those which attain a $90 \%$ flatness during the pre-production run are marked with ' 1 ' while other bins are marked ' 0 '. This may be clarified as follows. We calculate the average histogram value for those bins which have been visited at least once, thus discarding the bins which are not visited at all. The flatness test (which needs each of the visited bins to have a histogram count at least equal to $90 \%$ of the average histogram) is then applied only to those bins and these are labelled with ' 1 '. In the 'production run' part of the rest of the simulation we check the flatness of only those bins which were marked ' 1 ' ignoring what is happening to the others. There is however, always a possibility, since the 'production run' generates many more microstates than that in the 'pre-production run', that larger areas in the $(E-S)$ space would get included in the initial 'visit-map' or those bins, once marked ' 0 ', would subsequently qualify for the label ' 1 '. But it is impossible to improve upon the accuracy of the work indefinitely and we decided to stick to the map we obtained during a reasonable amount of the 'pre-production run', ignoring what is happening to the discarded bins.

In addition to the two-dimensional random walk in the $(E-S)$ space we have also performed a number of other two-dimensional random walks. These involve the $(E-\rho(r))$ space where $\rho(r)$ is the correlation function defined in Eq. (8). We have done these only for the $L=160$ lattice, for $r$ ranging from 2 to 40 and the ensemble averages of $\rho(r)$ were evaluated for different temperatures using Eq. (3). 


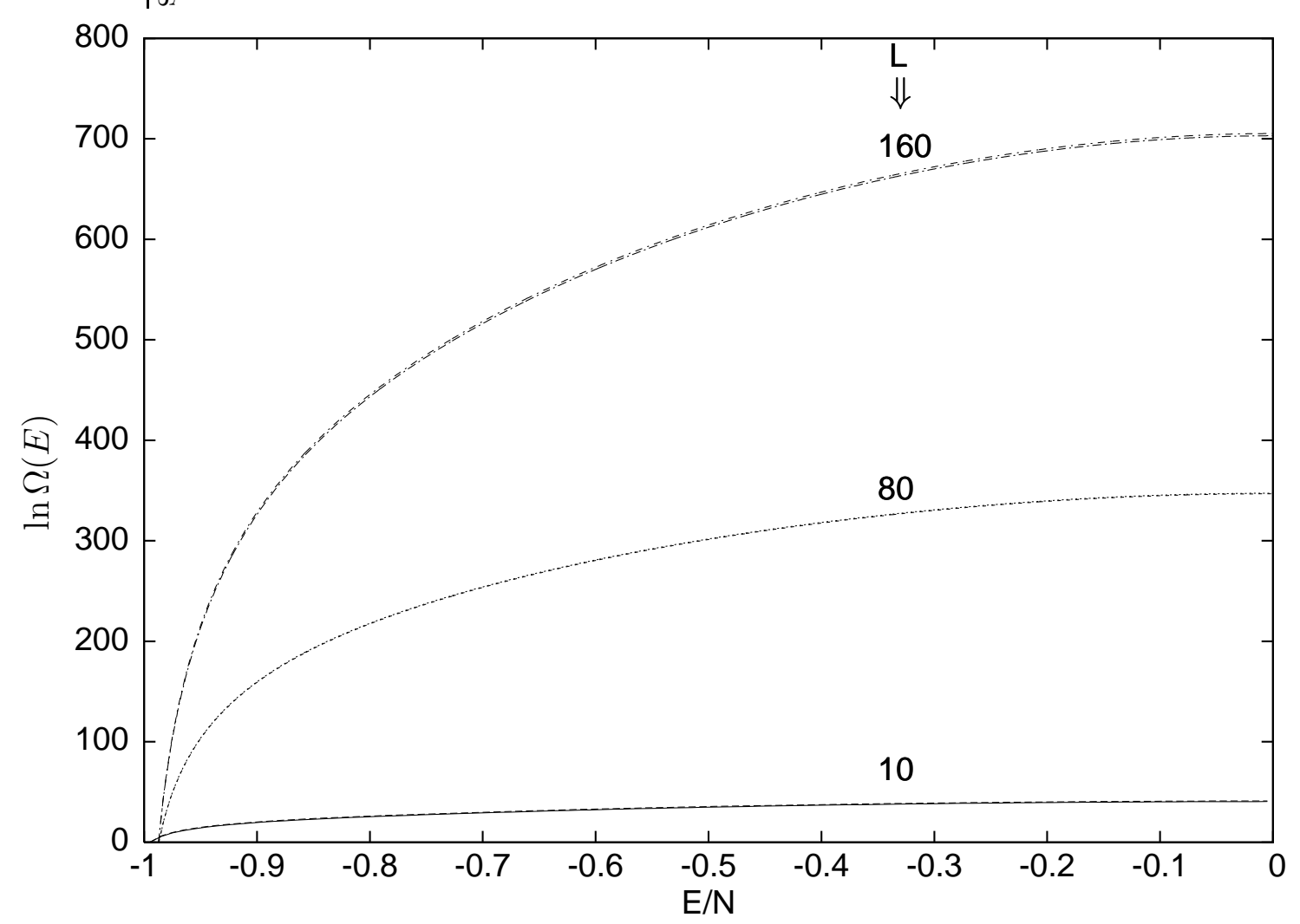

Figure 1: Logarithm of the density of states, $\ln \Omega(E)$, for the 1-d Lebwohl-Lasher model for $L=10,80$ and 160 obtained from 1-d and 2-d walks. In the resolution of the figure the data for 1-d and 2-d walks overlap.

\section{Results and Discussions}

We have performed MC simulations using the WL algorithm in linear spin chains of length $L$ where $L=10,20,40,80$ and 160 . All the results we present are results of a single simulation for each lattice size and we did not perform averaging of results over multiple simulations although this surely is expected to reduce the errors. In a simulation involving a continuous model one is confronted with the proper choice of the values of two parameters, $p$ and $d_{e}$ (defined in Sec. 3). The former determines the amplitude of the random rotation of a spin and the latter is the energy bin width. In the case of a two dimensional random walk, another parameter $d_{\phi}$, which represents the width of the order parameter or the correlation function bin is also to be chosen. We have set $d_{e}=0.1$ for all the work reported in this paper. $d_{\phi}$ was taken to be 0.01 for both order parameter and correlation function. The parameter $p$ was always 0.1 , except for the two- dimensional walk involving the order parameter, where 


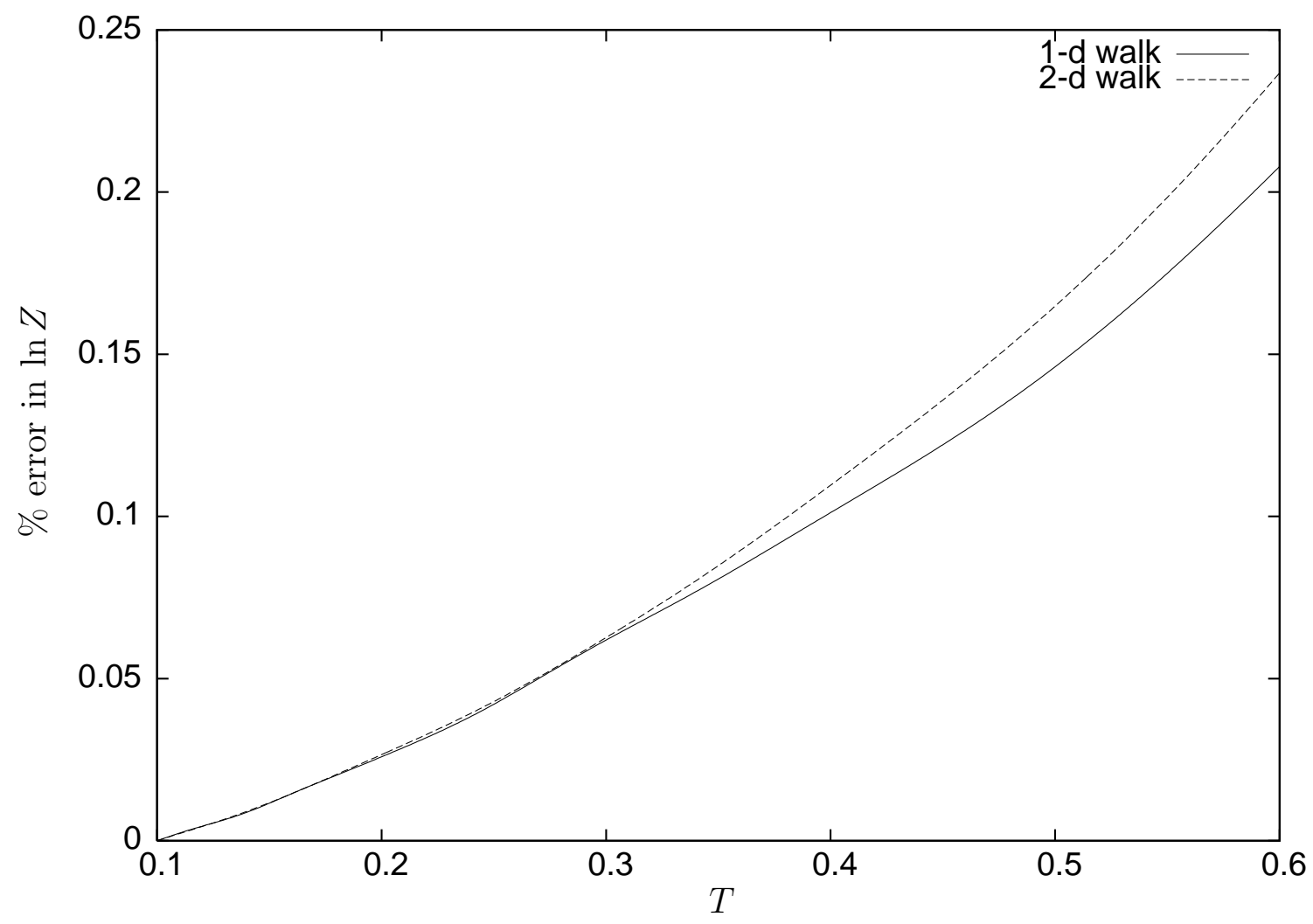

Figure 2: Percentage error in the logarithm of partition function, $\ln Z$, for 1-d and 2-d walks, plotted against temperature $T$, for the 1-d Lebwohl-Lasher model for $L=80$.

it was taken to be 0.2 . For larger values of $p$, the CPU time is less, but the results of the simulation (like the position and height of the specific heat curve, for instance) tend to depend strongly on $p$. For the values of $p$ in the neighbourhood of 0.1 , the results depend very weakly on $p$. This is presumably due to the fact that, a small change in the orientation of a spin (one at a time) results in a systematic and uniform sampling in the phase space, but as a result of greater correlation of the successive configurations generated and the consequent slow movement of the representative point in phase space, the computer time involved is greater. For the two-dimensional walk involving the order parameter, since a lot of CPU time is necessary, we have chosen $p=0.2$, to reduce the time involved. The bin widths for energy or other variables were so chosen that for about $50 \%$ of the configurations generated by the spin rotation procedure, new bins are visited. This procedure was found to be optimum, as an attempt to visit new bins more frequently, would result in missing a vast majority of the microstates which correspond to each bin. A small value of $p$, the rotation amplitude, is justifiable from the same point of view. A relatively large value of $p$ 
results in a poor sampling of the infinite number of closely spaced microstates contained in each bin and leads to poor results which tend to depend on the value of $p$, and consequently not in agreement with the exact results.

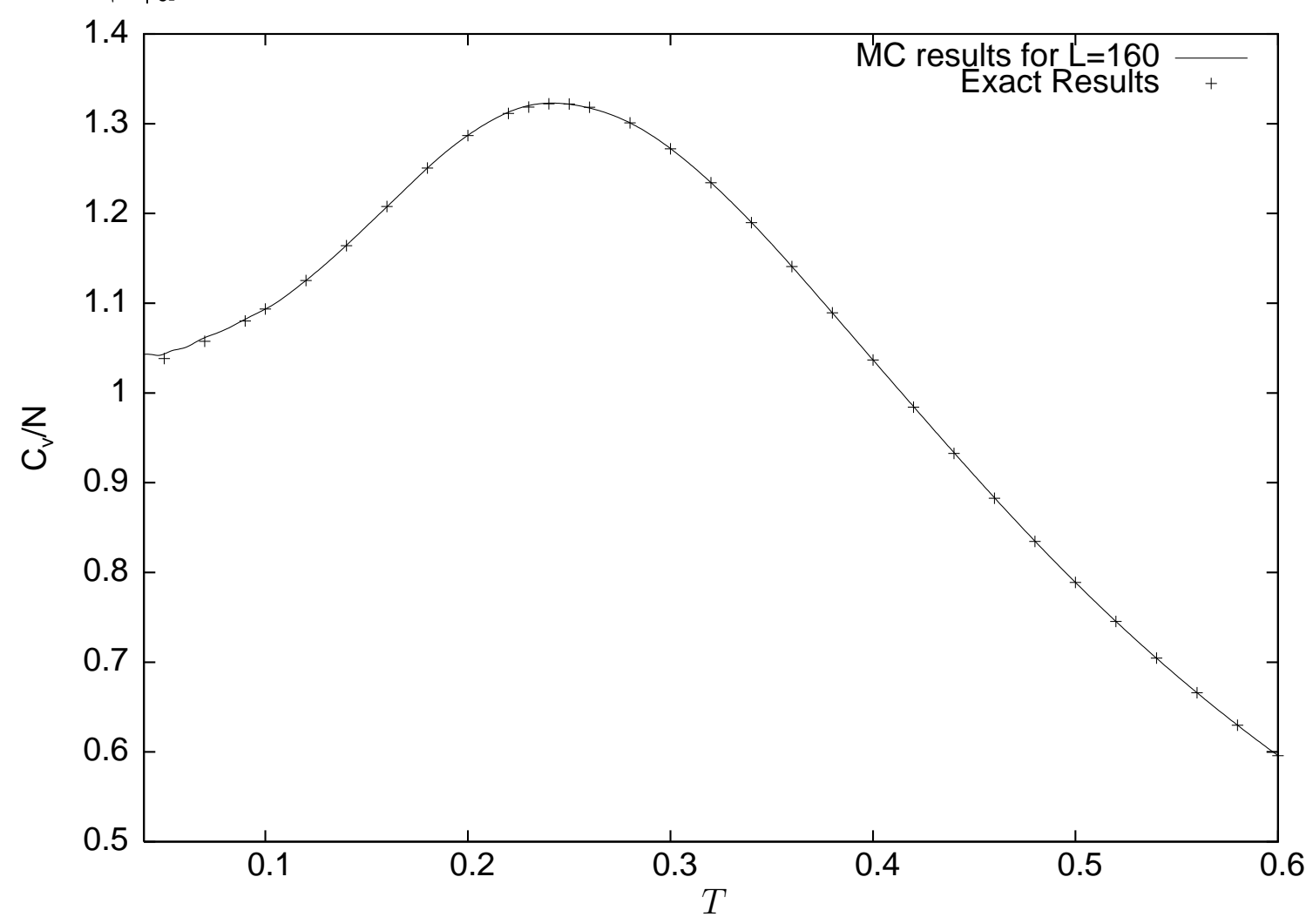

Figure 3: The specific heat per particle, obtained as a fluctuation quantity, plotted against temperature, for $L=160$ and compared with the exact result.

In Fig. 1 we have plotted the quantity $g(E)=\ln \Omega(E)$ as a function of the energy per particle for $L=10,80$ and 160 and the results obtained from one and two dimensional walks (in $E$ - $S$ space) have been compared. The system energy was always considered upto $E=0$. The lower limit of the energy for $L=80$ was -79 and for $L=160$, it was -158 , where the corresponding ground state energies are -80 and -160 . Thus, the visited energy range goes to a sufficiently low value to cover the entire range of interest though the small cut near the ground state was necessary, as it takes a huge time to sample these states for a relatively large lattice. The partition function, $Z$ was calculated from a knowledge of the density of states, and the percentage error in $\ln Z$, in comparison with the exact results, has been shown in Fig. 2 for both 1 -d and 2-d walk for the $L=80$ lattice. The error in $\ln Z$ slowly increases with temperature and, at the highest temperature we have investigated, $(\mathrm{T}=0.6)$, 
it is about $0.2 \%$; the error in $\ln Z$ available from $2-\mathrm{d}$ walk being marginally higher.

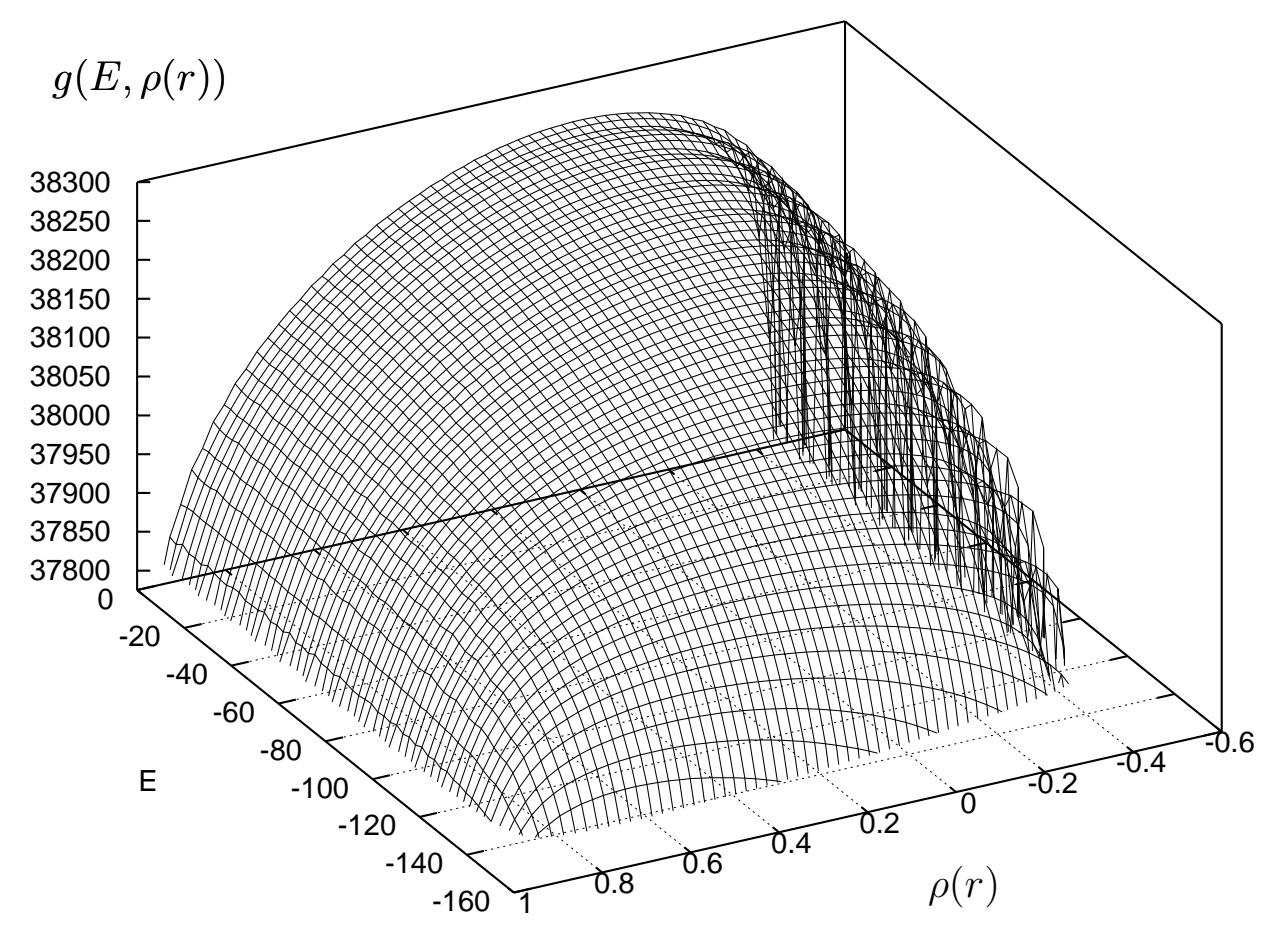

Figure 4: Three dimensional $g(E, \rho(r))$ surface plotted against energy and the correlation function for $r=5$.

The specific heat per particle has been plotted against temperature Fig. 3 for $L=160$ and compared with the exact results. In Fig. 4 we have depicted the density of statesurface, $\ln \Omega[E, \rho(r)]$ for $r=5$. The scalar order parameter $S$, defined in Eq. (7) has been plotted in Fig.5 against temperature for all lattice sizes. It may be recalled that the system is disordered at all finite temperature and one would expect $S=0$ for all values of $T$. For a given $T$ (including $T=0$ ), $S$ rapidly falls off with increase in system size, and in the thermodynamic limit will disappear altogether, as one would expect due to the finite size effect.

The correlation function $\rho(r)$ has been compared for the $L=160$ lattice using the twodimensional random walk. We have performed simulations for 12 values of $r$, ranging from 1 to 40, and these have been plotted against temperature in Fig. 6 where comparison has also been made with the exact results. It may be noted that, for each value of $r$, we had to run one simulation and the joint density of states were determined separately in each case. The 


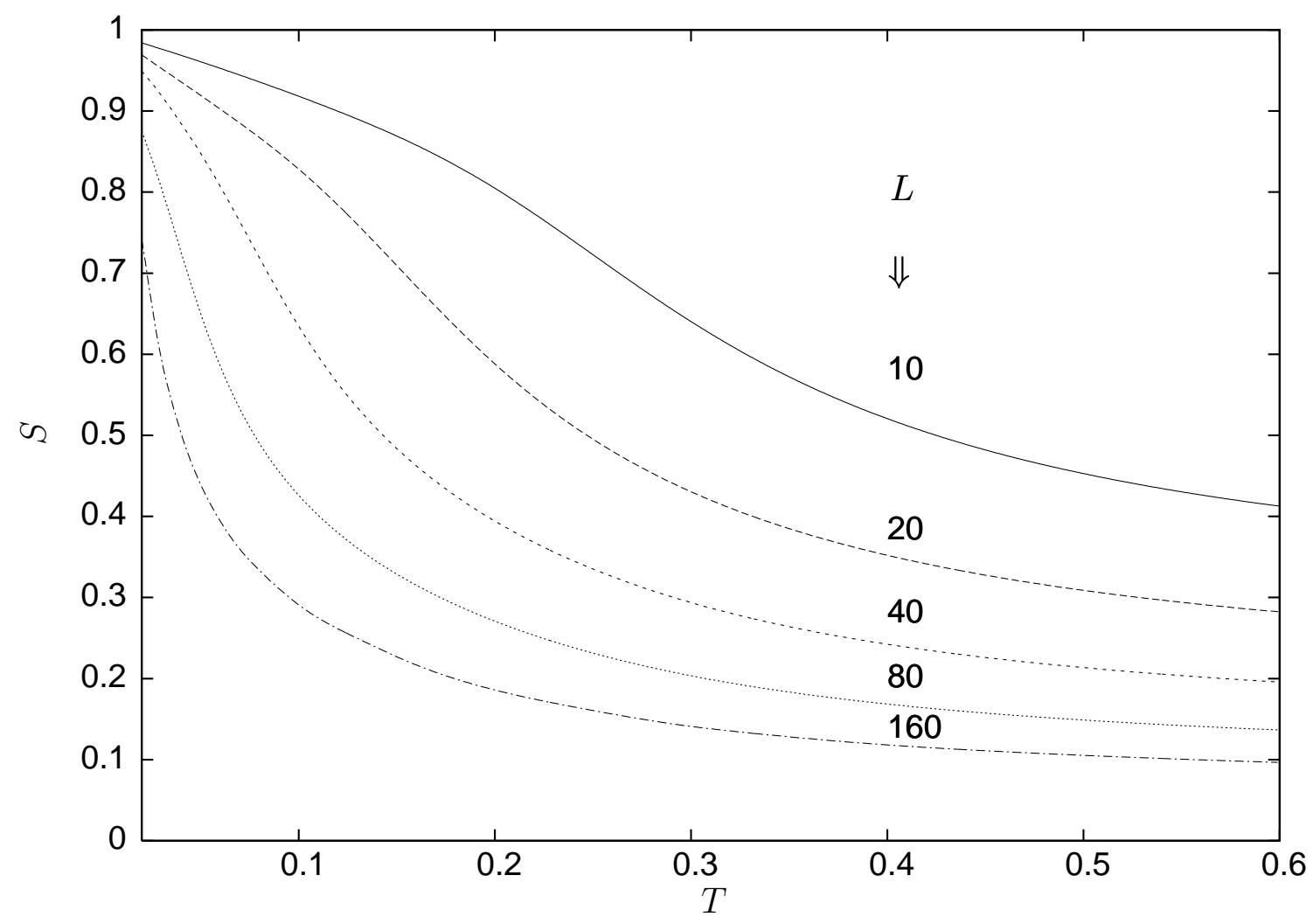

Figure 5: The temperature variation of orientational order parameter for different lattice sizes.

same data has also been shown in Fig. 7 where for three temperatures $\rho(r)$ has been plotted against $r$. As one would expect in a disordered system, the correlation dies off quickly with increase in $r$. For $r \rightarrow \infty$, the spins are uncorrelated and $\rho(r)$ should approach $S^{2}$. However, verification of this result from our simulation data will not make much sense in a disordered system. The CPU time necessary for the $L=160$ lattice for one-dimensional walk is 10.6 hours and the two-dimensional walk involving correlation function is 70.5 hours. For the two-dimensional $(E, S)$ walk the CPU time is 170 hours. The program was vectorized (i.e. the 'do' loops parallelized) between two $3.0 \mathrm{GHz}$ Xeon processors in a x226 IBM Server, automatically by the Intel Fortran Compiler, we used.

We end this section with a comment on the suggestions of Zhou and Bhatt [21] and Lee et. al. [22]. These authors have demonstrated that the requirement of a flat histogram is not necessary for the convergence of the density of states and it is sufficient to have $1 / \sqrt{\ln f}$ number of samplings of each macrostate for each iteration. They also have tested this with WL simulation in a two-dimensional Ising system (ferromagnetic as well as the 


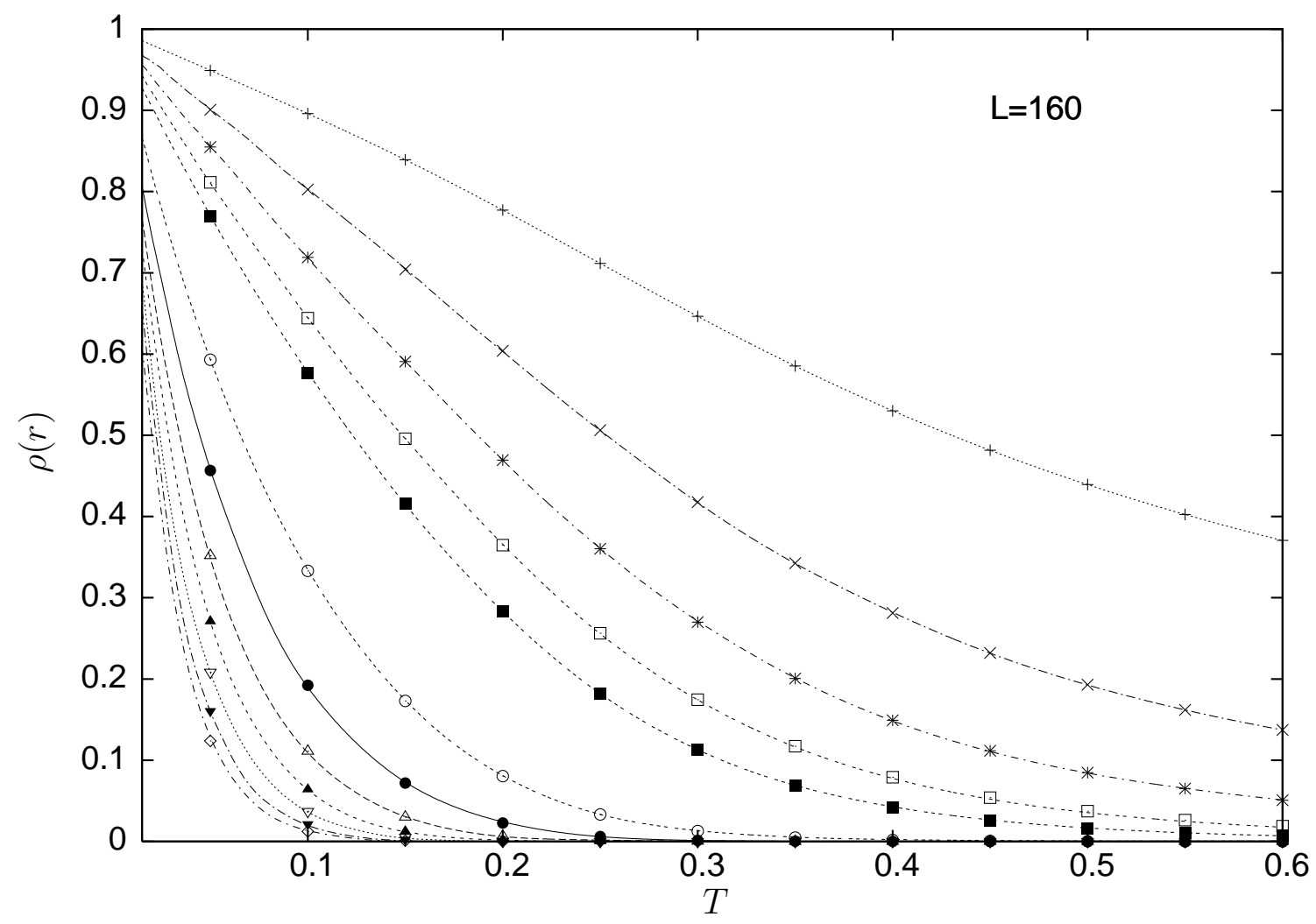

Figure 6: Variation of correlation function $\rho(r)$ with temperature $T$ for lattice size $L=160$. The points represented by different symbols are from the exact results obtained in Ref. [11, 12]. The curves are the results we obtained from the joint density of states. The values of $r$ taken are $1,2,3,4,5,10,15,20,25,30,35$ and 40 . The topmost curve is for $r=1$ and the lower curves are for other values of $r$ given in the sequence above and in ascending order of $r$.

fully frustrated model [22] ). We have also tried to implement this approach, but ended up with rather poor results for the two-dimensional random walk. We make a general comment that, for a system with a continuous energy spectrum, because of the infinite number of microstates associated with each bin, the minimum number of visits to each bin is expected to be dependent on the bin width and this needs a careful consideration for a two-dimensional random walk. On the other hand, the flatness criterion we have used, works satisfactorily for the determination of joint density of states in a continuous model, as our results demonstrate. 


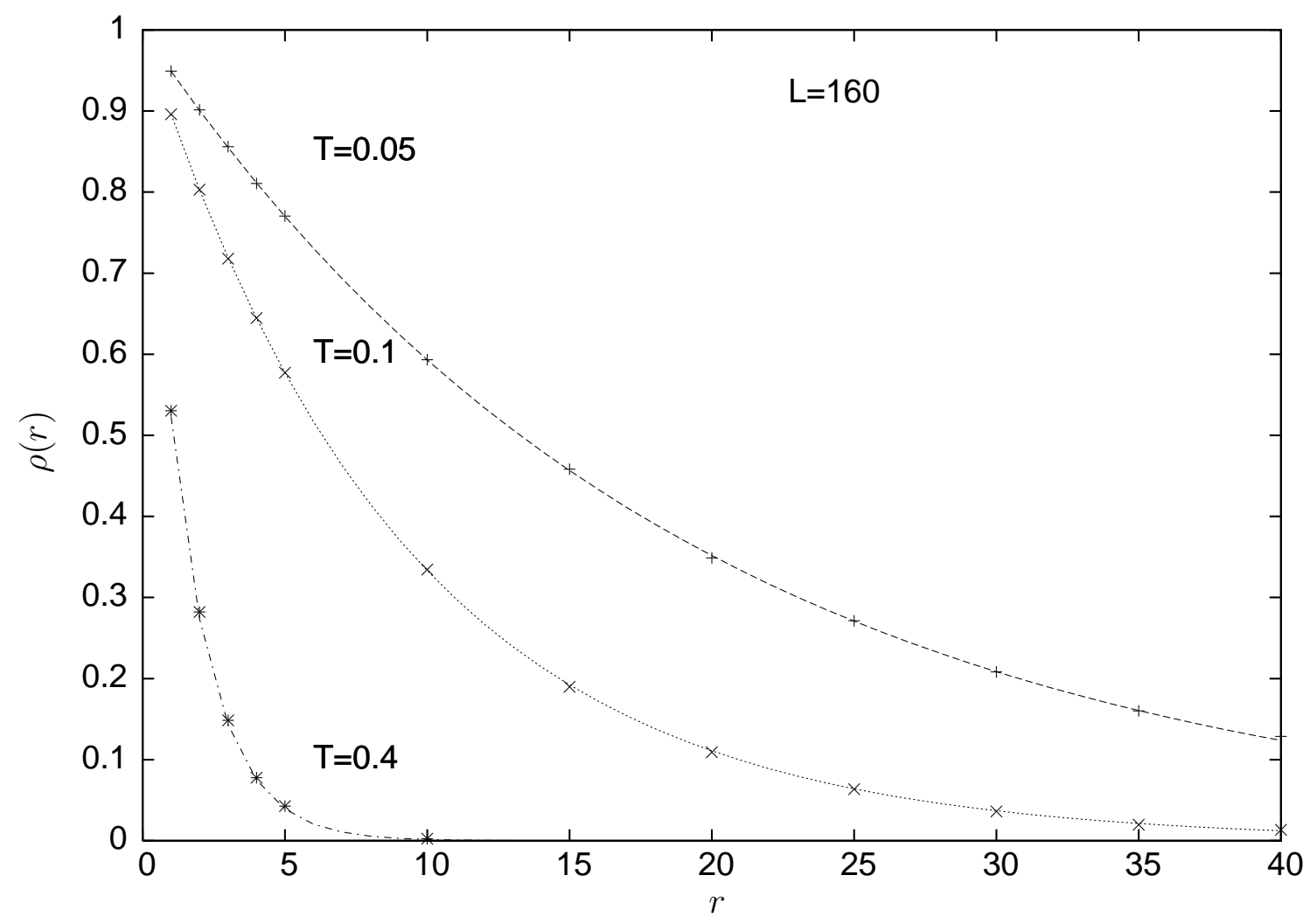

Figure 7: Correlation function as a function of $r$ at different temperatures.

\section{Conclusion}

The work we have presented in this communication, demonstrates that, using a flatness criterion one can successfully apply the WL algorithm to simulate a continuous model and can get reliable results for the joint density of states. We have attempted to provide a guideline for the proper choice of different parameters involved in the simulation of a continuous model and this may be useful for further work in such systems. However, the two-dimensional random walk in a continuous model becomes expensive in terms of the CPU time even for a system of moderate size, and it seems to be an impractical task to do this in a reasonably large lattice. We have also tried the local and global updating of histograms using Gaussian functions to generate the joint density of states, as has been proposed in the work of Zhou et. al. [5], but the results so far have not been encouraging. This needs further careful consideration. However, an improved or alternative algorithm would perhaps be more helpful. 


\section{Acknowledgment}

We acknowledge the receipt of a research grant No. 03(1071)/06/EMR-II from Council of Scientific and Industrial Research, India which helped us to procure the IBM x226 servers.

\section{References}

[1] F. Wang and D. P. Landau, Phys. Rev. Lett. 86 (2001) 2050; Phys. Rev. E 64 (2001) 056101.

[2] Q. L. Yan, R. Faller, and J. J. de Pablo, J. Chem. Phys. 116 (2002) 8745.

[3] M. S. Shell, P. G. Debenedetti and A. Z. Panagiotopoulos, J. Chem. Phys. 119 (2003) 9406.

[4] D. Jayasri, V. S. S. Sastry and K. P. N. Murthy, Phys. Rev. E 72 (2005) 036702.

[5] C. Zhou, T. C. Schulthess, S. Torbrgge and D. P. Landau, Phys. Rev. Lett. 96 (2006) 120201.

[6] P. Poulain, F. Calvo, R. Antoine, M. Broyer and Ph. Dugourd, Phys. Rev. E 73 (2006) 056704 .

[7] P. A. Lebwohl and G. Lasher, Phys. Rev. A 6 (1972) 426.

[8] W. Maier and A. Saupe, Z. Naturforsch, A 13 (1958) 564.

[9] W. Maier and A. Saupe, Z. Naturforsch, A 14 (1959) 882.

[10] W. Maier and A. Saupe, Z. Naturforsch, A 15 (1960) 287.

[11] P. A. Vuillermot and M. V. Romerio, J. Phys. C 6 (1973) 2922.

[12] P. A. Vuillermot and M. V. Romerio, Commun. Math. Phys., 41 (1975) 281.

[13] See for instance P. M. Chaikin And T. C. Lubensky, Principles of Condensed Matter Physics, Cambridge University Press, Cambridge, 1995.

[14] N. D. Mermin and H. Wagner, Phys. Rev. Lett. 17 (1966) 1133. 
[15] H. Kunz and G. Zumbach, Phys. Rev. B 46 (1992) 662.

[16] E. Mondal and S. K. Roy, Phys. Lett. A 312 (2003) 397.

[17] S. Dutta and S. K. Roy, Phys. Rev. E 70 (2004) 066125.

[18] C. Chiccoli, P. Pasini and C. Zannoni, Liq. Cryst. 3 (1988) 363.

[19] M. Abramowitz and I. Stegun, A Handbook of Mathematical functions, Dover, New York, 1970.

[20] A. Troster and C. Dellago, Phys. Rev. E 71 (2005) 066705.

[21] C. Zhou and R. N. Bhatt, Phys. Rev. E 72 (2005) 025701.

[22] H. K. Lee, Y. Okabe and D. P. Landau, Comp. Phys. Comm., 175 (2006) 36. 